\title{
Nonlinear screening theory of the Coulomb glass
}

\author{
Sergey Pankov \\ Laboratoire de Physique Théorique, Ecole Normale Supérieure, \\ 24 Rue Lhomond, 75231 Paris Cedex 05, France \\ Vladimir Dobrosavljević \\ Department of Physics and National High Magnetic Field Laboratory, Florida State University, Tallahassee, FL 32306
}

(Dated: August 14, 2018)

\begin{abstract}
A nonlinear screening theory is formulated to study the problem of gap formation and its relation to glassy freezing in classical Coulomb glasses. We find that a pseudo-gap ("plasma dip") in a single-particle density of states begins to open already at temperatures comparable to the Coulomb energy. This phenomenon is shown to reflect the emergence of short range correlations in a liquid (plasma) phase, a process which occurs even in the absence of disorder. Glassy ordering emerges when disorder is present, but this occurs only at temperatures roughly an order of magnitude lower. Our result demonstrate that the formation of the "plasma dip" at high temperatures is a process distinct from the formation of the Efros-Shklovskii (ES) pseudo-gap, which in our model emerges only within the glassy phase.
\end{abstract}

The interplay of interactions and disorder remains one of the most important open problems in condensed matter physics. These effects are most dramatic in disordered insulators, where the pioneering work of Efros and Shklovskii (ES) 1] emphasized the fundamental significance of the long-ranged nature of Coulomb interactions. This work presented convincing evidence that at $T=0 \mathrm{a}$ soft "Coulomb gap" emerges in the single-particle density of states (DOS) which, in arbitrary dimension $d$, reads

$$
g(\varepsilon) \sim \varepsilon^{d-1} .
$$

¿From a general point of view this result is quite surprising. It indicates a power-law distribution of excitation energies, i.e. the absence of a characteristic energy scale for excitations above the ground state. Such behavior is common in models with broken continuous symmetry, where it reflects the corresponding Goldstone modes, but is generally not expected in discrete symmetry models, such as the one used by ES. Here, it may reflect unusually strong frustration behavior inherent to Coulomb interactions in presence of disorder.

Indeed, the ES model seems to display several glassy features characterized by a large number of meta-stable states and slow relaxation, as clearly seen in many simulations [2, 3, 4, 5], and even in some experiments [7]. Interestingly, a precursor of the gap begins to appear [4, 6] already at relatively high temperatures, while glassy ordering emerges only at much lower $T$. Similar behavior has been identified even in absence of randomness [8]. Is the physics of the Coulomb gap thus related or is it unrelated to the glassy features of the system? The close connection between the two phenomena was recently demonstrated [9] for a mean-field model of interacting disordered electrons in the limit of large coordination, but the issue remains unresolved for Coulomb systems in finite dimensions where the ES theory applies.
To address these important issues in a controlled and precise fashion, we make the following observation which is the main physical point of this letter. We stress that the principal ES result - the emergence of a power-law spectrum - is not specific to low dimensions! Its physical origin and its relation to high temperature anomalies can, therefore, be investigated by the theoretical approaches controlled in the limit of high spatial dimensions. Such a theory for the Coulomb glass (CG) model is presented in this letter. Our main conclusions are as follows: (1) A non-universal pseudo-gap in the DOS (we call it the "plasma dip") begins to emerge at temperatures of the order of the Coulomb energy. It reflects strong short range correlations in the Coulomb plasma, a feature that is most pronounced in absence of disorder [8], but is unrelated to the Coulomb gap of ES. We obtain simple analytical results that in quantitative detail describe the temperature evolution of the plasma dip, in excellent agreement with all existing simulations. (2) The high temperature plasma (fluid) phase becomes unstable to ergodicity breaking at temperatures typically ten times lower, as the system enters a glassy state. We argue that a true ES pseudo-gap emerges only within the glassy (nonergodic) phase, and that its scale-invariant form reflects the marginal stability of such a glassy state.

Nonlinear screening theory. The simplest many-body approach to Coulomb systems is the well-known DebyeHuckel theory, which provides a linear screening description equivalent to a Gaussian approximation for the plasmon mode. This formulation, however, fails badly at low temperatures, where nonlinear effects lead to strong correlations in the plasma phase. Even worse, such a Gaussian theory is unable to describe glassy freezing even in the well-understood mean-field limit corresponding to infinite range interactions. To overcome these difficulties, we use the simplest theory of nonlinear screening given by the classical limit of the so-called extended dynamical 
mean-field theory [10], which also describes the leading order nontrivial correlations in the limit of large coordination. In this approach, the environment of a given site is approximated by free collective modes (plasmons in our case), the dispersion of which is self-consistently determined. In recent work, a version of this method has been successfully applied to the problem of self-generated glassiness [11] in systems with frustrated phase separation [12] without disorder. Here, we apply it to the classical Coulomb glass model [1] given by the Hamiltonian

$$
H=\sum_{i} \phi_{i} n_{i}+\frac{1}{2} \sum_{i j} V_{i j}\left(n_{i}-K\right)\left(n_{j}-K\right),
$$

where $n_{i}=0,1$ is the electron occupation number, and $\phi_{i}$ is a Gaussian distributed random potential of variance $W^{2}$. We express the Coulomb interaction $V_{i j}=\varepsilon_{0} / r_{i j}$ in units of the nearest-neighbor repulsion $\varepsilon_{0}$, and the intersite distance $r_{i j}$ in units of the lattice spacing. We adopt the following notation throughout the paper. For vectors and matrices in the replica space we use bold font and the hat symbol correspondingly. In the replica symmetric ansatz (RS) a matrix $\hat{O} \equiv\left\{O_{c}, O\right\}$ is parameterized by its connected part $O_{c}$ and its off-diagonal part $O$. Thus for the density density correlator we use $\hat{q} \equiv\{\chi, q\}$. Thermal and disorder averages are denoted as $\langle O\rangle_{T}$ and $[O]_{\text {dis }}$ respectively, and $\langle O\rangle \equiv\left[\langle O\rangle_{T}\right]_{\text {dis }}$.

To derive the desired self-consistency equations we average over disorder using the standard replica method [9], and use a cavity construction [9, 10], integrating out the degrees of freedom on all sites except the considered one. The resulting contribution to the local effective action is computed in the Gaussian approximation, giving a term of the form $-\frac{1}{2} \delta \mathbf{n} \hat{\Delta} \delta \mathbf{n}$, where $\delta \mathbf{n}=\mathbf{n}-\langle\mathbf{n}\rangle$. By requiring that the local density-density correlator $q^{\alpha \beta}=\left\langle\delta n^{\alpha} \delta n^{\beta}\right\rangle_{c}$ is correctly reproduced by the effective action, one obtains a self-consistency condition. We get

$$
\begin{aligned}
& q^{\alpha \beta}=\left\langle\delta n^{\alpha} \delta n^{\beta}\right\rangle_{c S_{e f f}}, \\
& S_{e f f}=-\frac{1}{2} \delta \mathbf{n} \hat{\tilde{\Delta}} \delta \mathbf{n} \\
& \hat{q}=\sum_{k}\left(\hat{q}^{-1}+\hat{\Delta}-\beta V_{k}\right)^{-1}
\end{aligned}
$$

where $\tilde{\Delta}^{\alpha \beta}=\Delta^{\alpha \beta}+\beta^{2} W^{2}$, and $V_{k}$ is the Fourier transform of the interaction potential. In case of the RS solution $\hat{\tilde{\Delta}} \equiv\left\{\Delta_{c}, \beta^{2} W_{e f f}^{2}\right\}$, where $W_{\text {eff }}=\sqrt{W^{2}+\beta^{-2} \Delta}$ is the renormalized disorder.

Fluid solution. To examine the evolution of the system in the high temperature phase we first examine the $\mathrm{RS}$ solution. In the $n \rightarrow 0$ replica limit we get

$$
q=\frac{1}{4} \int D[x] \tanh ^{2}\left(\frac{1}{2} x \beta W_{e f f}\right)
$$

where $D[x] \equiv(2 \pi)^{-1 / 2} \exp \left\{-x^{2} / 2\right\} \quad d x$. The selfconsistency condition becomes

$$
\begin{aligned}
& \chi+q=\frac{1}{4} \\
& \chi=\sum_{k}\left(\chi^{-1}+\Delta_{c}-\beta V_{k}\right)^{-1} \\
& q=\left(q \chi^{-2}-\Delta\right) \sum_{k}\left(\chi^{-1}+\Delta_{c}-\beta V_{k}\right)^{-2}
\end{aligned}
$$

The Eqs. (45) can easily be solved numerically for the parameters $\chi, q, \Delta_{c}, \Delta, W_{e f f}$, to calculate the density of states (DOS) function $g(\epsilon)$, to examine the stability of the RS solution, and to compute the entropy $S$. The above equations are written for the half filled case in absence of charge ordering. Generalization to uniformly ordered phases is a standard procedure [13], where the ordering transition is signaled by a divergence in $\chi_{k}=\left(\chi^{-1}+\Delta_{c}-\beta V_{k}\right)^{-1}$ at the ordering vector $k=Q$. For simplicity, most of our results are written in the homogeneous phase at half filling and that will be assumed unless stated otherwise.

Density of states. For the CG model, the single particle DOS (tunneling DOS) function $g(\epsilon)$ is simply given by the distribution of the local fields (energies) $\epsilon_{i}=\partial H / \partial n_{i}$ :

$$
g(\epsilon)=\sum_{i}\left\langle\delta\left(\epsilon-\epsilon_{i}\right)\right\rangle
$$

Integrating out all sites except one, we derive an expression for $g(\epsilon)$ in terms of the local effective field $\hat{\tilde{\Delta}}$. The final result for the $\mathrm{RS}$ solution reads:

$$
\begin{aligned}
g(\epsilon)= & \int D[x] \frac{\beta}{\sqrt{2 \pi \Delta_{c}}} \frac{\cosh \frac{1}{2} \beta \epsilon}{\cosh \frac{1}{2} x \beta W_{\text {eff }}} \\
& \times \exp \left\{-\frac{1}{2 \Delta_{c}}\left[\frac{1}{4} \Delta_{c}^{2}+\left(\beta \epsilon+x \beta W_{e f f}\right)^{2}\right]\right\} .
\end{aligned}
$$

In the cavity method langauge 14 this result can be interpreted as the Gaussian distribution of the cavity fields (different from the local fields) of variance $W_{\text {eff }}$, modified by the the (Onsager) self reaction term representing the plasma correlations.

We get insight into the behavior of the DOS by considering some analytically solvable limits. At $T \rightarrow 0$, the DOS remains finite at the Fermi level, though it can be exponentially small for weak disorder. This shows that the fluid (RS) solution does not capture the physics of the true ES gap, which emerges only within the glassy phase. On the other hand, as long as the RS solution is stable, a large "plasma dip" may develop, but it will have no direct relation to the glassy physics or the ES gap. It reflects strong short-range correlations in the Coulomb plasma (fluid) phase, which are suppressed at large disorder. Here, the RS DOS reduces to the bare disorder 
distribution. In the opposite limit of vanishing disorder $W_{\text {eff }} \rightarrow 0$ the DOS expression simplifies and for an arbitrary filling reads:

$$
\begin{aligned}
g(\epsilon)=\frac{\beta}{\sqrt{2 \pi \Delta_{c}}} & \exp \left(-\frac{\frac{1}{4} \Delta_{c}^{2}+\beta^{2} \epsilon^{2}}{2 \Delta_{c}}\right) \\
\times & {\left[\cosh \frac{1}{2} \beta \epsilon-(2 K-1) \sinh \frac{1}{2} \beta \epsilon\right] . }
\end{aligned}
$$

To support the validity of our formulation, we compare our analytical results with available numerical simulations. In Fig. 11 (top panel), we examine the situation studied in Ref.[6], where calculations were done for a 3D CG on a cubic lattice, for a set of temperatures in the fluid phase. The lowest temperature is very near the glass transition temperature (see our phase diagram, Fig. 2). As we can see, our theory captures in surprisingly quantitative detail the formation of the "plasma dip" in the fluid phase. In the past, this phenomenon has often been confused with the formation of the true ES gap which, as we argue below, only emerges within the glassy phase. Finally, we test limits of our theory by computing the DOS for the 2D CG in absence of disorder. Even in this extreme case, we reproduce semi-quantitatively exact numerical results of Ref. [8].

Glassy ordering. To examine the stability of the fluid phase to glassy ordering, we examine the Baym Kadanoff (BK) functional $\Gamma_{B K}[\hat{q}]$. This is a functional of the correlator $\hat{q}$, which yields the exact equations of motion at the saddle point, where it coincides with the exact free energy. To obtain our self-consistency conditions, a local approximation [13] is made on the two particle irreducible part of $\Gamma_{B K}$. In this formulation, the stability of our fluid RS solution can be obtained by a standard replica symmetry breaking (RSB) analysis 15] of the BK functional at the saddle point. The corresponding RSB instability criterion takes the form

$$
\frac{1}{\left[\chi_{i i}^{2}\right]_{d i s}}-\frac{1}{\left[\chi_{i i}\right]_{d i s}^{2}}+\frac{1}{\sum_{j}\left[\chi_{i j}\right]_{d i s}^{2}}=0 .
$$

Here, $\chi_{i j}$ is the density-density correlation function computed for a fixed realization of disorder, i.e. $\chi_{i j}=$ $\left\langle\delta n_{i} \delta n_{j}\right\rangle_{T}-\left\langle\delta n_{i}\right\rangle_{T}\left\langle\delta n_{j}\right\rangle_{T}$. The left hand side of Eq. (9) is nothing but $1 / \sum_{j}\left[\chi_{i j}^{2}\right]_{d i s}$, the inverse of the glass susceptibility, a quantity which diverges at the transition. In terms of the RS solution the RSB condition reads

$$
\frac{q}{\Delta}=\frac{1}{16} \int D[x] \cosh ^{-4}\left(\frac{1}{2} x \beta W_{\text {eff }}\right) .
$$

As an illustration, we present results for the CG on a 3D cubic lattice, and in Fig. [2 we plot the corresponding phase diagrams obtained by numerically solving our selfconsistency conditions. At small disorder and temperature $T \approx .95$ (which is in satisfactory agreement with the

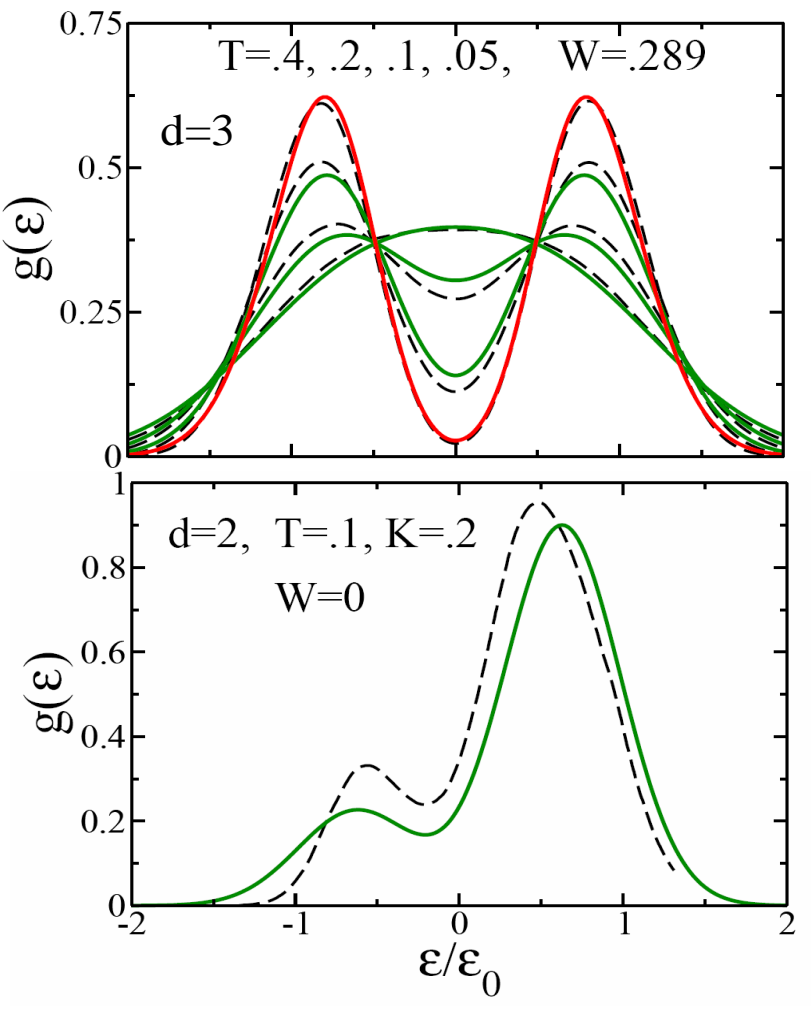

FIG. 1: Our analytical predictions for the single-particle density of states (full lines) are found to be in excellent quantitative agreement with simulation results (dashed lines), with no adjustable parameters. Shown are results for the three dimensional case studied in from Ref. [6], corresponding to $W=1 /(2 \sqrt{3})$, and temperatures $T=0.4,0.2,0.1,0.05$ (top panel), and the two dimensional model of Ref. [8], corresponding to $W=0, T=0.1, K=0.2$. All lines correspond to the plasma phase, while $T=0.05$ is very close to the glass transition temperature.

exact value 17] $\left.T_{c}=.129\right)$ the system enters the charge ordered phase. phase. Stronger disorder suppresses the charge ordering, and the system can exist either in a liquid phase (at higher temperature) or in the glass phase (at lower $T$ ). The liquid is separated from the glass by the RSB line, also known as the Almeida-Thouless [15] line. We emphasize that the ordering temperature we predict is roughly an order of magnitude smaller then the Coulomb energy, in remarkable quantitative agreement with all available simulation results $[\underline{3}, 4,[4,[8]$.

This interesting fact can be traced down to the screening of the Coulomb interaction. Indeed, the overall energy scale characterizing the screened Coulomb potential $V_{s c r}(r)=\varepsilon_{0} \exp \left\{-r / \ell_{s c r}\right\} / r$ is roughly an order of magnitude smaller then the bare Coulomb energy. The corresponding screening length $\ell_{s c r}=\left[\left(\chi^{-1}+\Delta_{c}\right) /\left(\beta \varepsilon_{0}\right)\right]^{1 / 2}$ (shown for $W=(2 \sqrt{3})^{-1}$ in the inset of Fig. 2) decreases (albeit weakly) with temperature, and remains short throughout the fluid (RS) phase. This observation also makes it clear why the true ES gap cannot emerge 


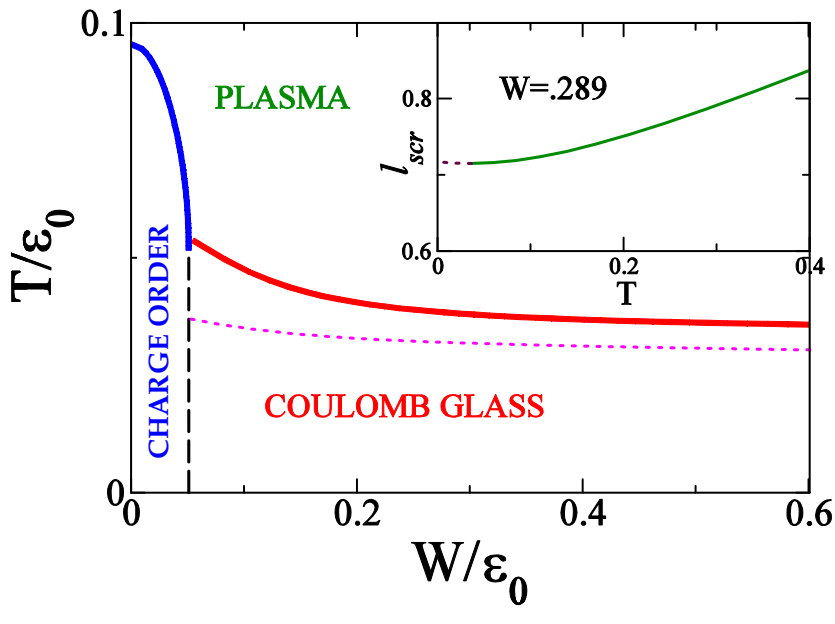

FIG. 2: 3D Coulomb glass phase diagram. The full horizontal line indicates the RSB instability and the dotted line shows where the RS entropy turns negative. The screening length in the inset is plotted for the same disorder and the range of temperatures (fluid phase), as in Fig 1

in absence of glassy ordering. The screening mechanism remains operative throughout the (ergodic) fluid phase, and thus the long-range character of the Coulomb interaction, which is crucial for the ES argument remains inoperative. In contrast, within the glass phase, following the arguments from Ref. [9], we expect the relevant zero-field cooled compressibility to decrease and vanish at $T=0$. This mechanism opens a route for the screening to be suppressed at low temperatures, and the true ES gap to emerge.

To provide further evidence of the instability of the fluid phase to glassy ordering, we also calculate the entropy in the fluid (RS) solution, which takes the form

$$
\begin{aligned}
S=\int D[x] \ln & {\left[2 \cosh \left(\frac{1}{2} x \beta W_{\text {eff }}\right)\right] } \\
& +\frac{1}{2} \sum_{k} \ln \chi_{k}-\frac{1}{2} \ln \chi-2 \chi \beta W_{\text {eff }} .
\end{aligned}
$$

It is well known that for standard mean-field glass models, the RS replica theory predicts negative entropy at $T=0$, while the lower bound for the glass transition temperature can be set where the RS entropy changes sign. It is easy to show from Eq. (11) that our RS entropy proves strictly negative at $T=0$ as well. In Fig. 2 we also plot (dashed line) a lower bound for the RSB instability line where the RS entropy turns negative, providing further evidence that the fluid phase cannot survive down to $T=0$.

The glass phase and the Efros-Shklovskii gap. In this letter we do not explicitly examine the RSB solution of our model. Nevertheless, we follow arguments similar to those of Ref. 9], and use the large disorder asymptotics of the glass transition line to determine the powerlaw form of the $T=0$ Coulomb gap in the glassy phase. At $W \gg \varepsilon_{0}$, we find

$$
T_{G} \sim \varepsilon_{0}^{1+\frac{1}{\gamma}} W^{-\frac{1}{\gamma}}
$$

where the exponent $\gamma$ is identical to that predicted by ES for the $T=0$ DOS in the CG

$$
g(\epsilon) \sim \varepsilon_{0}^{-1-\gamma} \epsilon^{\gamma} .
$$

For an interaction of a general power law form $V(r) \sim$ $\varepsilon_{0} / r^{a}$, the ES argument predicts $\gamma=(d-a) / a$ in arbitrary dimension $d$. The asymptotic regime, however, sets in at larger values of disorder, not shown in the Fig.(2).

Let us explain the importance of Eq. (12). We have seen that for $W \neq 0$, the DOS remains finite in the RS fluid phase, so a true ES pseudo-gap can emerge only due to glassy ordering. For another electron glass model, work of Ref. [9] has established that the emergence of a true pseudo-gap at $T=0$ directly follows from the marginal stability of the glassy state, and that its form also determines the large disorder asymptotics of $T_{G}(W)$. Given the close similarity of our mean-field equations for the CG model to those examined in Ref. 9], we expect the same mechanism to apply here as well. Using the expected form of the ES gap, we can estimate the glass transition temperature as the energy scale $E_{\text {gap }}$ characterizing the "width" of the gap that opens in the low temperature phase. We find $E_{\text {gap }} \sim \varepsilon_{0}^{1+\frac{1}{\gamma}} W^{-\frac{1}{\gamma}}$, coinciding with Eq. (12). This result presents strong evidence in favor of the close relation between glassy ordering and the emergence of the ES gap.

In conclusion, we have formulated a simple many-body theory that is able to clarify the relation between the finite temperature formation of the Coulomb gap and the emergence of glassy ordering in disordered Coulomb systems in finite dimensions. This nonlinear screening approach is flexible enough to allow for future extensions to quantum models [9, 16], and to study the role of Anderson and Mott localization [18] in Coulomb systems.

We thank G. Biroli, A. Georges, D. Grempel, M. Muller, D. Popović, B. Shklovskii, T. Vojta, and G. Zimanyi for useful discussions. This work at FSU was supported by the NSF grant DMR-0234215. SP acknowledges support from the CNRS, France. After this work has been completed, the authors became aware of the closely related calculation of M. Muller and L. B. Ioffe (cond-mat/0406324), where complementary results consistent with our findings were presented.

[1] A. L. Efros and B. I. Shklovskii, J. Phys. C 8, L49 (1975).

[2] J. H. Davies, et al., Phys. Rev. Lett. 49, 758 (1982).

[3] A. Möbius, et al., Phys. Rev. B 45, 11568 (1992).

[4] E. R. Grannan and C. C. Yu, Phys. Rev. Lett. 71, 3335 (1993). 
[5] D. Grempel, Europhys. Lett. 66, 854 (2004).

[6] M. Sarvestani, et al., Phys. Rev. B 52, R3820 (1995).

[7] A. Vaknin et al., Phys. Rev. Lett. 81, 669 (1998); ibid., 84, 3402 (2000); S. Bogdanovich and D. Popović, Phys. Rev. Lett. 88, 236401 (2002).

[8] A. L. Efros, Phys. Rev. Lett. 68, 2208-2211 (1992).

[9] A. A. Pastor, and V. Dobrosavljević, Phys. Rev. Lett. 83, 4642 (1999).

[10] J. L. Smith, and Q. Si, Phys. Rev. B 61, 5184 (2000); R. Chitra, and G. Kotliar, Phys. Rev. Lett. 843678 (2000).

[11] A. V. Lopatin, and L. B. Ioffe, Phys. Rev. B 66, 174202 (2002).
[12] H. Westfahl, et al., Phys. Rev. B 68, 134203 (2003); S. $\mathrm{Wu}$, et al., cond-mat/0305404

[13] R. Chitra, and G. Kotliar, Phys. Rev. B 63, 115110 (2001) ; S. Pankov, et al., Phys. Rev. B 66, 045117 (2002).

[14] M. Mezard, et al., Europhys. Lett. 1, 77 (1986).

[15] J. R. L. de Almeida, D.J. Thouless, J. Phys. A 11, 983 (1978)

[16] F. Epperlein et al., Phys. Rev. B 56, 5890 (1997).

[17] A. Möbius and U. K. Rößler, cond-mat/0309001

[18] V. Dobrosavljević, et al., Phys. Rev. Lett. 90, 016402 (2003). 